\title{
Intra-abdominal EWSR1/FUS-CREM-rearranged malignant epithelioid neoplasms: two cases of an emerging aggressive entity with emphasis on misleading immunophenotype
}

\author{
Abbas Agaimy ${ }^{1,2}$ (D) Robert Stoehr ${ }^{1,2} \cdot$ Mike Otto $^{3} \cdot$ Jan Hinrich Bräsen ${ }^{4} \cdot$ Nicole Pfarr $^{5} \cdot$ Björn Konukiewitz $^{5}$. \\ Atsuko Kasajima $^{5} \cdot$ Arndt Hartmann $^{1,2} \cdot$ Günter Klöppel $^{5}$
}

Received: 29 April 2021 / Revised: 31 May 2021 / Accepted: 7 June 2021 / Published online: 6 July 2021

(C) The Author(s) 2021

\begin{abstract}
CREB family (CREB 1, ATF 1, and CREM) gene fusions are defining markers in diverse mesenchymal neoplasms (clear cell sarcoma, angiomatoid fibrous histiocytoma, and others). However, neoplasms harboring EWSRI-CREM/FUS-CREM fusions are rare and poorly characterized. We describe two cases (55-year-old male with $7.5 \mathrm{~cm}$ renal mass and 32-yearold female with $5.5 \mathrm{~cm}$ mesenteric mass) illustrating their misleading immunophenotypes. Histologically, both showed eosinophilic and focally clear epithelioid cells arranged into sheets, nests, and trabeculae. Immunohistochemistry showed ALK, EMA, and AE1/AE3 immunoreactivity suggesting $A L K$-rearranged renal cell carcinoma (Case 1) and coexpression of keratin, EMA, synaptophysin, and chromogranin-A, suggesting neuroendocrine neoplasm (Case 2). Targeted RNA sequencing revealed EWSR1-CREM (Case 1) and FUS-CREM (Case 2) fusions. These cases add to the spectrum of CREM fusion-positive intra-abdominal epithelioid neoplasms. Their unusual immunophenotype and unexpected sites represent major pitfalls, underline a wide differential diagnosis, and emphasize the value of molecular testing in correctly diagnosing them.
\end{abstract}

Keywords RCC · ALK · EWSR1 - CREB · CREM · FUS · Primary renal sarcoma · Mesentery · Neuroendocrine · Paraganglioma

\section{Introduction}

Gene fusions involving the Ewing Sarcoma Breakpoint Region 1 gene (EWSRI on 22q) and one of the CREB family genes (CREB1, ATF1, and CREM) have been increasingly

Abbas Agaimy

abbas.agaimy@uk-erlangen.de

1 Institute of Pathology, Friedrich-Alexander University Erlangen-Nürnberg (FAU), University Hospital Erlangen (UKER), Erlangen, Germany

2 Comprehensive Cancer Center Erlangen-EMN (CCC ER-EMN), Erlangen, Germany

3 Medical Center of Histology, Cytology and Molecular Diagnostics Trier LLC (GmbH), Trier, Germany

4 Institute of Pathology, Hannover Medical School, Hannover, Germany

5 Institute of Pathology, Technical University Munich, Munich, Germany recognized in soft tissue and visceral neoplasms with significant phenotypic and demographic diversity [1]. The major representatives of this category are clear cell sarcoma (CCS) of tendon and aponeuroses (mainly EWSRI-ATF1 fusions), malignant gastrointestinal neuroectodermal tumor (mainly EWSRI-CREBI fusions), angiomatoid fibrous histiocytoma (mainly EWSRI-CREBI fusions), and clear cell carcinomas of the head and neck (EWSRI-ATFl fusions) [1]. As a group, these neoplasms differ significantly in morphology, immunophenotypes, and behavior, ranging from low-grade indolent (e. g., angiomatoid fibrous histiocytoma) to aggressive (e.g., CCS) [1]. Within the CREB fusion family, CREM-rearranged neoplasms are rare. They include subsets of CCS, angiomatoid fibrous histiocytomas, and clear cell carcinoma of the head and neck [2]. We describe two CREM-rearranged intra-abdominal malignant epithelioid neoplasms (one renal and one mesenteric) displaying confusing immunophenotypes: expression of ALK and keratin (Case 1) and keratin and neuroendocrine markers (Case 2). In both, diagnosis was only made after NGS testing uncovered the underlying fusions. 


\section{Materials and methods}

Cases were retrieved from our consultation files. Case 2 was identified among 25 mesenchymal/non-epithelial neoplasms in a cohort of 346 neuroendocrine mimics (Kasajima et al., submitted). Immunohistochemistry (IHC) was performed on 3- $\mu$ m sections using a fully automated system ("Benchmark XT System," Ventana Medical Systems Inc.) and the following antibodies: keratin (AE1/AE3, 1:40, Zytomed), vimentin (V9, 1:100, Dako), PAX8 (rabbit polyclonal, 1:50, Cell Marque), CK7 (OV-TL, 1:1000, Biogenex), CK5 (XM26, 1:50, Zytomed), TTF-1 (8G7G3/1, 1:500, Zytomed), ERG (EPR3864, prediluted, Ventana Medical Systems), CD31 (JC70A, 1:20, Dako), CD10 (56C6, 1:20, Dako), p63 (SFI-6, 1:100, DCS), desmin (D33, 1:250, Dako), smooth muscle actin (1A4, 1:200, Dako), S100 protein (polyclonal, 1:2500, Dako), CD34 (BI-3C5, 1:200, Zytomed), CD30 (Ber-H2, 1:40, Zytomed), MUC4 (EP256, 1:500, Epitomics), TLE1 (polyclonal, 1:200, Santa Cruz), STAT6 (sc-621, 1:1000, Santa Cruz), SMARCB1/INI1 (MRQ27, 1:50, Zytomed), synaptophysin (MQR40, 1:1, Ventana/ Roche), chromogranin-A (LK2H10, 1:500, Thermo Fisher), SDHB (polyclonal, 1:200, Sigma-Aldrich), TFE3 (clone MRQ-37, 1:100, Cell Marque), and MUC1 (MRQ17, 1:50, Cell Marque) according to the manufacturer instructions. FFPE tissue from Case 2 was studied by electron microscopy (LEO 912, Zeiss, Oberkochen, Germany) with digital image device (TRS Tröndle, Moorenweis, Germany) according to routine methods.

\section{Molecular testing}

RNA-based NGS was performed as described previously [3]. ALK/EWSRI (Case 1) and FUS/TFE3 (Case 2) FISH analyses were performed using ZytoLight ${ }^{\circledR}$ SPEC Dual Color Break Apart Probes (ZytoVision, Bremerhaven, Germany) designed to detect translocations involving these genes, respectively. Case 2 was tested for $S D H B$ mutations using the methods described previously [4].

\section{Results}

\section{Case histories}

\section{Case 1}

A 55-year-old male underwent radical nephrectomy for a renal mass. His medical history was negative for other malignancies. He is currently diagnosed with metastatic disease in the pelvis 23 months from initial diagnosis.

\section{Case 2}

A 32-year-old female without other primary malignancy presented with a mesenteric mass that was resected together with the adjacent small bowel segment. She presented 8 months later with peritoneal, pleural, and lymph node metastases. Currently (13 months from initial diagnosis), she is alive with extensive progressive disease under palliative chemotherapy.

\section{Pathological findings}

\section{Case 1}

The nephrectomy specimen contained a well-demarcated $7.5 \mathrm{~cm}$ renal medullary mass, distinct from the renal pelvis without extrarenal extension. Histological examination showed medium-sized epithelioid cells disposed into communicating nests and trabeculae within a prominent fibrous stroma, entrapping glomeruli and tubules (Fig. 1A, B). The nuclei were moderately vesicular with small inconspicuous nucleoli surrounded by granular eosinophilic to clear cytoplasm (Fig. 1C). A few rhabdoid cells were seen. Necrosis was absent. $<4$ mitoses/10 HPFs were found. IHC showed diffuse expression of vimentin, EMA, AE1/AE3 (Fig. 1D), cytoplasmic ALK (Fig. 1E), and variably (40\% of cells) MUC4 (Fig. 1F). All other markers listed above including PAX8 (Fig. 1D inset) were negative. SMARCB1 expression was retained.

\section{Case 2}

The tumor measured $5.5 \mathrm{~cm}$ and was intra-mesenteric distinct from the gut wall. Histological examination showed medium-sized epithelioid cells forming well vascularized sheets with variably eosinophilic, clear (Fig. 2A, B), rhabdoid, and spindled (Fig. 2C) cell morphology lacking a distinct stroma. Focal pseudotrabecular structures within desmoplastic stroma were noted (Fig. 2D). Fifteen mitoses were observed in $10 \mathrm{HPFs}$. Hemorrhagic necrosis was present. IHC revealed strong positivity for AE1/AE3, CK18, synaptophysin (Fig. 2E), chromogranin-A (Fig. 2F) and MUC1, and loss of SDHB. TFE3 showed diffuse moderate reactivity. SMARCB1 expression was retained. Repeated electron microscopy revealed no cytoplasmic membrane bound neurosecretory granules.

\section{Molecular findings}

With a provisional diagnosis of ALK + renal cell carcinoma (RCC), ALK FISH failed to show rearrangement or copy 
Fig. 1 Representative histological images of Case 1 show epithelioid cells disposed into communicating irregular nests within fibrous stroma (A) with focal entrapment of the glomeruli (B). C At high power, the epithelioid morphology is seen; note the variably granular eosinophilic to clear cytoplasm. D AE1/AE3 reveals mainly paranuclear dot-like pattern (note the strong expression in entrapped tubules; main image). D, inset, PAX8 highlights entrapped tubules, but the tumor cells are negative. E Strong cytoplasmic ALK expression is seen. F MUC4 is variably positive
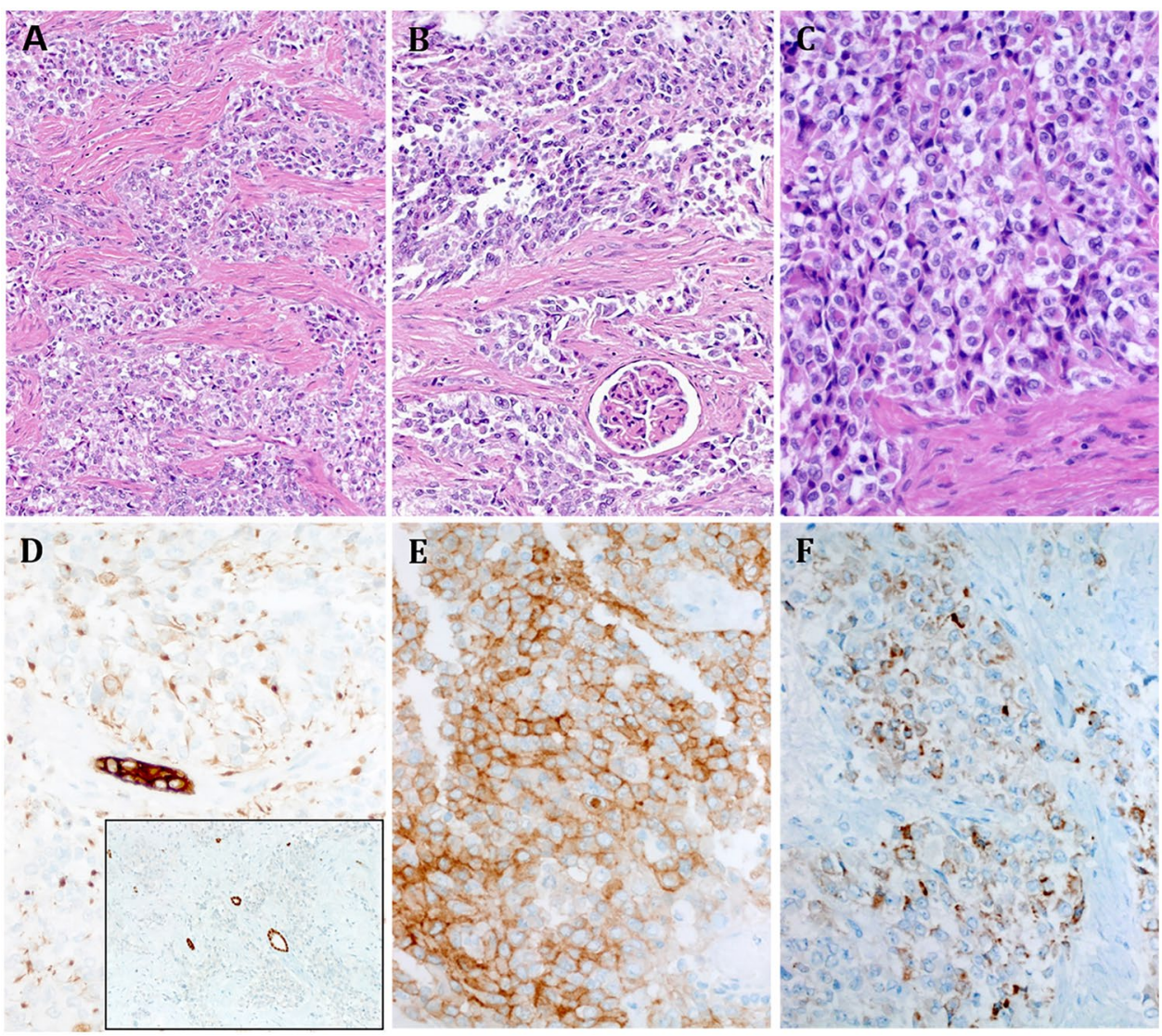

number gains in Case 1. Targeted RNA sequencing revealed a EWSRI-CREM fusion (Fig. 3), which was confirmed by EWSR1 FISH. Case 2 (mesenteric tumor) showed a FUSCREM fusion (Fig. 3) which was also confirmed by FUS FISH. NGS (Case 2) revealed no SDHB mutation (which was suggested by the immunohistochemical loss of the protein).

\section{Discussion}

With increasing use of targeted RNA NGS in routine surgical pathology practice, the numbers and variants of gene fusions detected in neoplasms have been increasing, and several new molecularly defined entities are emerging. EWSR1/FUS-CREM fusions were recognized recently in a group of unclassified epithelioid mesenchymal neoplasms showing predilection for intra-abdominal organs, not fitting any known EWSRI-CREB-rearranged entity [1, 2]. We herein expand the topographic and phenotypic features of these emerging neoplasms and highlight increasing pitfalls associated with them. Including our cases, 12 EWSRl/ FUS-CREM-positive intra-abdominal epithelioid neoplasms have been reported $[2,5,6]$. Affected sites were omentum/ mesentery (4), stomach (2), kidney (2), pelvic organs (2), adrenal (1), and unspecified site (1). Seven tumors harbored EWSR1-CREM and five FUS-CREM fusions [2, 5, 6]. Very few examples occurred at extra-abdominal soft tissue (chest wall) or visceral (lung) sites [2, 7]. These neoplasms possess a definite malignant potential with propensity for peritoneal recurrences and nodal and distant metastasis $[2,5,6]$.

The morphology of these tumors seems distinctive. They all displayed monotonous epithelioid morphology with variable cytoplasmic clearing and sparse fibrous stroma. Most cases expressed keratin and EMA [2, 5, 6]. ALK was positive in one (intra-abdominal [2]), synaptophysin in two (chest wall and lung [2,7]), and MUC4 in two (chest wall and gastric [2,6]) previous cases. We add the expression of ALK, MUC4, and synaptophysin in one case each and report novel chromogranin-A expression in one.

Although the MUC4-positive gastric tumor was diagnosed as sclerosing epithelioid fibrosarcoma (SEF), the depicted histology suggests similarity to the current cases with variable clear cell epithelioid pattern, lacking characteristic sclerosis of SEF [6]. Another reported EWSR1-CREMpositive SEF was not illustrated [8]. Including our cases, $3 / 5$ tumors tested positive for MUC4. This finding is relevant given that MUC4 expression and presence of EWSR 1 fusion by FISH are otherwise considered diagnostic of SEF in the context of epithelioid mesenchymal neoplasms [9]. 
Fig. 2 Case 2 showed mediumsized epithelioid cells forming solid sheets lacking a distinct stroma with variable cytoplasmic clearing (A, B). C Focal areas displayed rhabdoid or spindle-shaped morphology. D A pseudotrabecular pattern within desmoplastic stroma was seen focally. Immunohistochemistry revealed strong expression of synaptophysin $(\mathbf{E})$ and chromogranin-A (F)

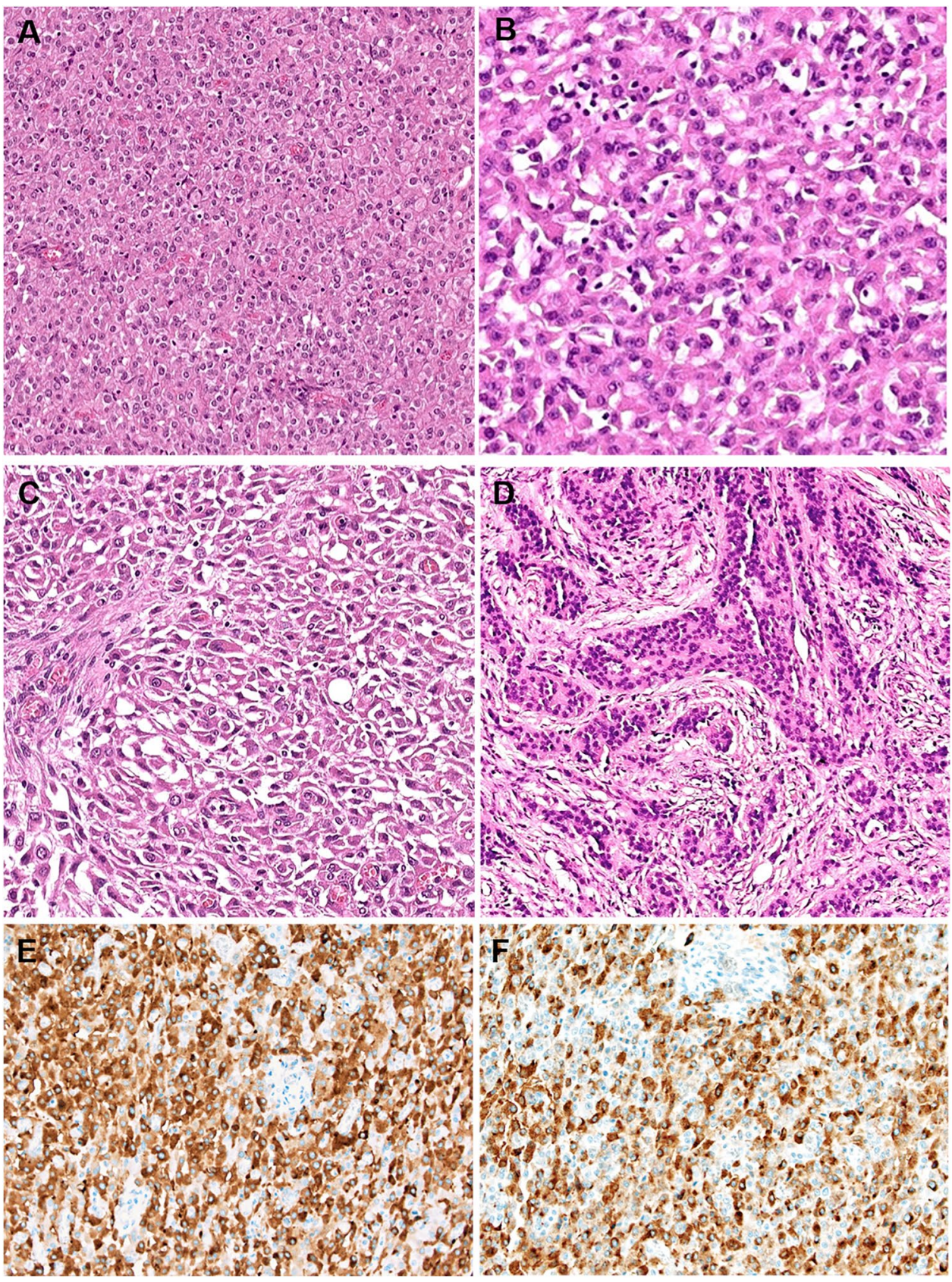

However, distinction of the two entities seems justified to address their potentially different biology. Most SEFs are driven by EWSR1-CREB3L1 and rarely by FUS-CREB3L1 fusions [1].

Our renal case expands the spectrum of EWSRI-rearranged renal sarcomas and represents the second EWSRICREM fusion-positive renal case [5]. Moreover, it represents true pitfall as detection of ALK in eosinophilic RCC strongly suggests an $A L K$-rearranged RCC [10]. Indeed, this was our initial diagnosis. The absence of PAX8 suggests alternative diagnoses and justifies further molecular testing in such a case. ALK expression is common in several translocation sarcomas including angiomatoid fibrous histiocytoma [11] and rhabdomyosarcoma [12]. The molecular basis of ALK immunoreactivity observed in diverse fusion-associated soft tissue tumors seems heterogeneous and associates with specific types of fusions (e.g., EWSRI/FUS-TFCP2 and EWSRI but not MEIS1-NCOA2 and other fusions [11, 12]). While the ALK protein expression in alveolar rhabdomyosarcoma and neuroblastoma correlates frequently with $A L K$ copy number gains, none of angiomatoid fibrous histiocytoma showed this feature [11]. ALK might represent a downstream target of EWSR1 and PAX-FOXO1 fusion proteins, but this remains speculative $[11,12]$. 


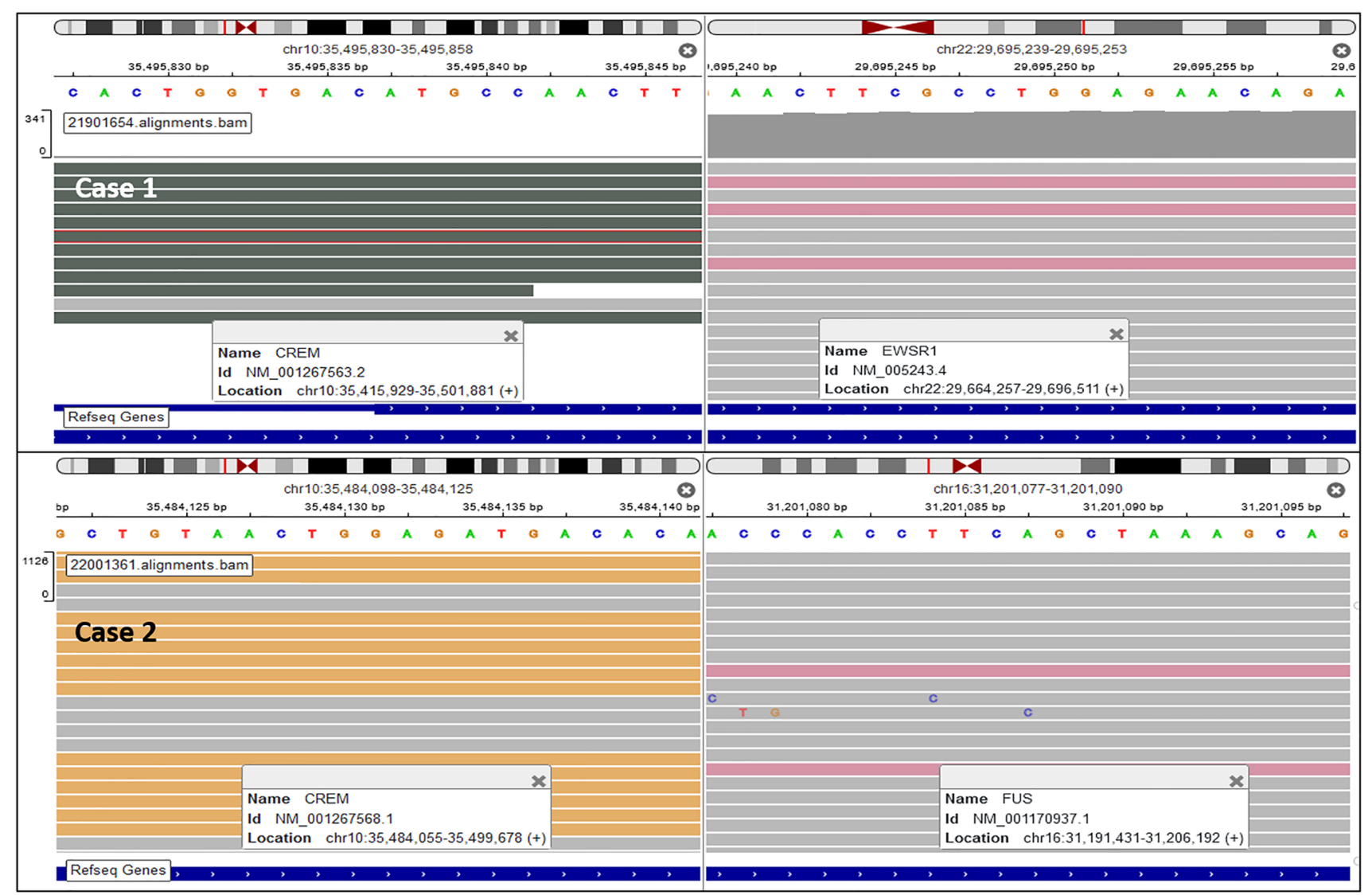

Fig. 3 IGV split-screen view of read alignments of the identified CREM-EWSR1 (Case 1) and CREM-FUS (Case 2) fusion events. Shown are the breakpoints in the CREM (left) and the EWSR1/FUS (right) genes, respectively. Alignments whose mate pairs are mapped

Regarding Case 2, it is noteworthy that it labeled not only for synaptophysin but also for chromogranin-A, a combination that we encountered only rarely in mesenchymal neoplasms with neuroendocrine features (data not shown). Since chromogranin-A normally resides in the membrane of neurosecretory granules, we studied the tumor tissue by electron microscopy to search for neurosecretory granules but could not identify any. We conclude that chromogranin-A protein associates with other cell organelles than secretory granules in this neoplasm. Loss of SDHB in this case remained unexplained and represents a further confusing factor with neuroendocrine neoplasms. Finally, aberrant or equivocal TFE3 expression noted in Case 2 and in a previous case [7] may suggest a TFE3-rearranged neoplasm. This is particularly important in renal cases as rare Xp11-translocation RCC may harbor EWSR 1-TFE3 fusions and be indistinguishable from EWSRI/FUS-CREM neoplasms by FISH alone [13]. All these observations underline the morphological and immunophenotypic heterogeneity of EWSRI/FUS-CREM fusion driven mesenchymal neoplasms. to the fusion sequence on the other chromosome are colored in dark green/pink for CREM-EWSR1 and ocher/pink for CREM-FUS. All other alignments are colored gray

In summary, we report two intra-abdominal malignant epithelioid mesenchymal neoplasms carrying the recently described EWSR1-CREM and FUS-CREM fusions. Besides their epithelioid morphology, these tumors displayed unusual misleading immunophenotypes. Recognition of these findings should help to avoid misinterpretation as SEF, $A L K$-rearranged RCC, and neuroendocrine carcinoma. The cases highlight the value of precise genotyping in these challenging lesions to ovoid misdiagnoses.

Author contribution AA, GK: conception and design of the work; acquisition, analysis, and interpretation of data; and drafting the MS and revising it critically for important intellectual content and scientific integrity.

AA, RS, MO, JHB, NP, BK, AK, AH, and GK: acquisition, analysis, and interpretation of data and reading and revising the MS critically for important intellectual content and scientific integrity. All authors have read and approved the final manuscript.

Funding Open Access funding enabled and organized by Projekt DEAL. 


\section{Declarations}

Ethics approval Samples were used in accordance with ethical guidelines for the use of retrospective tissue samples provided by the local ethics committee of the Friedrich-Alexander University ErlangenNuremberg (ethics committee statements 24.01.2005 and 18.01.2012).

Conflict of interest The authors declare no competing interests.

Open Access This article is licensed under a Creative Commons Attribution 4.0 International License, which permits use, sharing, adaptation, distribution and reproduction in any medium or format, as long as you give appropriate credit to the original author(s) and the source, provide a link to the Creative Commons licence, and indicate if changes were made. The images or other third party material in this article are included in the article's Creative Commons licence, unless indicated otherwise in a credit line to the material. If material is not included in the article's Creative Commons licence and your intended use is not permitted by statutory regulation or exceeds the permitted use, you will need to obtain permission directly from the copyright holder. To view a copy of this licence, visit http://creativecommons.org/licenses/by/4.0/.

\section{References}

1. Thway K, Fisher C (2019) Mesenchymal tumors with EWSR1 gene rearrangements. Surg Pathol Clin 12:165-190

2. Yoshida A, Wakai S, Ryo E, Miyata K, Miyazawa M, Yoshida KI, Motoi T, Ogawa C, Iwata S, Kobayashi E, Watanabe SI, Kawai A, Mori T (2019) Expanding the phenotypic spectrum of mesenchymal tumors harboring the EWSR1-CREM fusion. Am J Surg Pathol 43:1622-1630

3. Agaimy A, Tögel L, Haller F, Zenk J, Hornung J, Märkl B (2020) YAP1-NUTM1 gene fusion in porocarcinoma of the external auditory canal. Head Neck Pathol 14:982-990

4. Konukiewitz B, Jesinghaus M, Steiger K, Schlitter AM, Kasajima A, Sipos B, Zamboni G, Weichert W, Pfarr N, Klöppel G (2018) Pancreatic neuroendocrine carcinomas reveal a closer relationship to ductal adenocarcinomas than to neuroendocrine tumors G3. Hum Pathol 77:70-79

5. Argani P, Harvey I, Nielsen GP, Takano A, Suurmeijer AJH, Voltaggio L, Zhang L, Sung YS, Stenzinger A, Mechtersheimer G, Dickson BC, Antonescu CR (2020) EWSR1/FUS-CREB fusions define a distinctive malignant epithelioid neoplasm with predilection for mesothelial-lined cavities. Mod Pathol 33:2233-2243

6. Memon RA, Granada CNP, Patel C, Manne U, Heslin MJ, Gbolahan OB, Harada S, Diffalha SA (2020) Gastric sclerosing epithelioid fibrosarcoma harboring a rare FUS-CREM fusion. Int J Surg Pathol :1066896920961174

7. Komatsu M, Sakai Y, Nishikubo M, Tane S, Nishio W, Kajimoto K, Hirose T (2020) EWSR1-CREM fusion in pulmonary mesenchymal neoplasm showing distinctive clear cell morphology. Pathol Int 70:1020-1026

8. Arbajian E, Puls F, Antonescu CR, Amary F, Sciot R, DebiecRychter M, Sumathi VP, Järås M, Magnusson L, Nilsson J, Hofvander J, Mertens F (2017) In-depth genetic analysis of sclerosing epithelioid fibrosarcoma reveals recurrent genomic alterations and potential treatment targets. Clin Cancer Res 23:7426-7434

9. Doyle LA, Wang WL, Dal Cin P, Lopez-Terrada D, Mertens F, Lazar AJ, Fletcher CD, Hornick JL (2012) MUC4 is a sensitive and extremely useful marker for sclerosing epithelioid fibrosarcoma: association with FUS gene rearrangement. Am J Surg Pathol 36:1444-1451

10. Kuroda N, Trpkov K, Gao Y, Tretiakova M, Liu YJ, Ulamec M, Takeuchi K, Agaimy A, Przybycin C, Magi-Galluzzi C, Fushimi S, Kojima F, Sibony M, Hang JF, Pan CC, Yilmaz A, Siadat F, Sugawara E, Just PA, Ptakova N, Hes O (2020) ALK rearranged renal cell carcinoma (ALK-RCC): a multi-institutional study of twelve cases with identification of novel partner genes CLIP1, KIF5B and KIAA1217. Mod Pathol 33:2564-2579

11. Cheah AL, Zou Y, Lanigan C, Billings SD, Rubin BP, Hornick JL, Goldblum JR (2019) ALK expression in angiomatoid fibrous histiocytoma: a potential diagnostic pitfall. Am J Surg Pathol 43:93-101

12. Agaram NP, Zhang L, Sung YS, Cavalcanti MS, Torrence D, Wexler L, Francis G, Sommerville S, Swanson D, Dickson BC, Suurmeijer AJH, Williamson R, Antonescu CR (2019) Expanding the spectrum of intraosseous rhabdomyosarcoma: correlation between 2 distinct gene fusions and phenotype. Am J Surg Pathol 43:695-702

13. Fukuda H, Kato I, Furuya M, Tanaka R, Takagi T, Kondo T, Nagashima Y (2019) A novel partner of TFE3 in the Xp11 translocation renal cell carcinoma: clinicopathological analyses and detection of EWSR1-TFE3 fusion. Virchows Arch 474:389-393

Publisher's note Springer Nature remains neutral with regard to jurisdictional claims in published maps and institutional affiliations. 